\title{
40 cases of Single-incision laparoscopic surgery to totally extraperitoneal herniorrhaphy via umbilical incision under posterior rectus abdominis sheath
}

Xiaojun Wang ( $\sim$ xarwxj@126.com )

Ningbo University https://orcid.org/0000-0002-6264-8238

\section{Yizhong Zhang}

The Affiliated Hospital of Medical School of Ningbo University

Changlei Qi

The Affiliated Hospital of Medical school of Ningbo University

\section{Encheng Zhou}

The Affiliated Hospital of Medical School of Ningbo University

\section{Haiqiang Zhang}

The Affiliated Hospital of Medical School of Ningbo University

\section{Ting Fei}

The Affiliated Hospital of Medical School of Ningbo University

\section{Yongfeng Shuai}

The Affiliated Hospital of Medical School of Ningbo University

Haibo Jin

The Affiliated Hospital of Medical School of Ningbo University

\section{Research article}

Keywords: Transumbilical, Single-port device, Iconport, Totalllyextraperitoneal

Posted Date: August 15th, 2019

DOI: https://doi.org/10.21203/rs.2.12935/v1

License: (1) (1) This work is licensed under a Creative Commons Attribution 4.0 International License. Read Full License 


\section{Abstract}

Background Since 2008, the first case of Single-incision laparoscopic surgery totally extraperitoneal (SILS-TEP) was reported, there was more than 10 years history on SILS-TEP. Because of difficult to operation, SILS-TEP is popularized slowly. This study investigate the safety and feasibility of SILS-TEP under posterior rectus abdominis sheath. Methods A single $2.5 \mathrm{~cm}$ long cured incision was made along the lower umbilical border. A $5.0 \mathrm{~cm}$ size preperitoneal space was created by electrotome or blunt dissection under the posterior sheath and the linea alba. The silica gel sealing sleeve of Iconport single port device was crammthed under the posterior rectus abdominis sheath, then the preperitoneal space was broadened toward lower abdomen in condition of $\mathrm{CO} 2$ gas inflation. Conventional laparoscopic instruments were used to complete the operation according to the operation procedure of laparoscopic total extraperitoneal herniorrhaphy. Results SILS-TEP hernia repair was successfully completed in 39 patients. One need an additional ports to complete totally extraperitoneal hernioplasty. Mean operative time was 73.8 minutes (range, 45 to 130 minutes) and 55.2 minutes (range, 34 to 95 minutes) in unilateral indirect hernia and in unilateral direct or femoral hernia, respectively. Mean operative time was 92.3 minutes (range, 65 to 150 minutes) in bilateral hernia. 3 seroma and 5 chronic pain were seen during the 1-6 months follow-up periods, all were treated conservatively. No other major complications. Mean post-operative hospital stay was 2.6 days. Conclusion The operation of SILS-TEP via umbilical incision under posterior rectus abdominis sheath is safe and feasible. The Iconport single-port device has potential advantages in SILS-TEP surgery. Trial registration: The study was registered with the Chinese Clinical Trial Registry (ChiCTR)(www.chictr.org.cn).Registration number:ChiCTR1900023056. Registration date: May 9, 2019.Retrospectively registered.

\section{Background}

Since 2008, the first case of Single-incision laparoscopic surgery totally extraperitoneal (SILS-TEP) was reported, there was more than 10 years history on SILS-TEP. Because of difficult to operation, SILS-TEP is popularized slowly. Our department started performing SILS-TEP in 2017, we use a self-made single

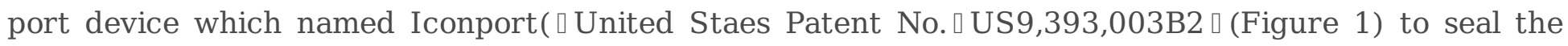
incision and complete SILS-TEP via umbilical incision under posterior rectus abdominis sheath. These 40 cases of SILS-TEP surgery are the experience from Jan ,2018 to Dec,2018.

\section{Methods}

Patient selection

We retrospectively analyzed 40 cases consecutive patients that underwent SILS-TEP between 1 January 2018 and 30 December 2018 at Affiliated Hospital of Medical School of Ningbo University. All patients with an inguinal hernia admitted to our institute were considered for SILS-TEP hernioplasty. Exclusion criteria were as follows: (1) an age of 20 years, (2) an acutely bowel incarcerated hernia, (3) previous 
retroperitoneal surgery, such as, for a recurrent hernia after laparoscopic hernioplasty or prostatectomy, or (4) a compromised cardiopulmonary function. In these cases, we performed open hernia repair. In this study, we analyzed only short-term outcomes. All surgeries were performed after obtaining informed consent from the patients, and the study was approved by our Institutional Review Board.

Surgical techniques

After gerneral anesthesia, the patient was placed in a supine position with the arm opposite the side of the hernia in adduction. A single $2.5 \mathrm{~cm}$ long cured incision was made along the lower umbilical border. A $5.0 \mathrm{~cm}$ size preperitoneal space was created by electrotome or blunt dissection under the posterior sheath and the linea alba. The silica gel sealing sleeve of Iconport single port device was crammthed under the posterior rectus abdominis sheath, then the preperitoneal space was broadened toward lower abdomen in condition of CO2 gas inflation(Figure 2). The preperitoneal space was dissected gradually using conventional straight and rigid types of laparoscopic instruments. Usually, the Retzius' space can be exposed easily(Figure 3) .When we begin to enlarge the preperitoneal space we should make the laparoscopic lens towards the right pubic tubercle of the patient and make sure that the peritoneum is able to identify gleamingly. When we expose the preperitoneal space adjacent Douglas'line, we should separate the preperitoneal tissue approaching superficial layer then the pectineal ligament and symphysis pubis can be identified in sequence. When we broaden the Bogros' space, outside of the inner ring was separated at first, then the laparoscopic operating instrument can insert into the Bogros' space. In cases of indirect hernias, the hernia sac was carefully isolated, freed from the spermatic cord, and reduced from the internal ring by gentle traction and dissection. There is different operative skills in different side of hernia during this course. In order to handle the laparoscopic instruments flexibly, we alternate use electric coagulation hook and laparoscopic forceps in different aisle in the panel. When the hernia sac was isolated and freed from the spermatic cord, we should choose the two adjacent aisle in the panel and keep the optical fiber of the lens between the hand shank of the two laparoscopic instruments in order to handle flexibly. When we finished to create the preperitoneal space, the whole myopectineal orifice was visualized, the hernia sac was visualized and freed from the spermatic cord, a $12 * 16 \mathrm{~cm}$ mesh (ETHICOH ULTRAPRO)was inserted and deployed to cover the whole myopectineal orifice(Figure 4,5,6,7,8). No fixation is required while for flat mesh. For bilateral hernia, the mesh is overlapped more than $1 \mathrm{~cm}$ over the midline to prevent recurrence. In case of female, the peritoneum was cut open at both side of uterine round ligament until its origination to keep it integrity. In case of peritoneum or the hernia sac tearing, if the size of peritoneum tearing was large, peritoneum was sutured, if the size was small, peritoneum was approximated using endo-clip. After completion of the operation, the preperitoneal space was deflated carefully to avoid displacing the mesh. The linea alba was closed with a 2-0 absorbable suture, and the skin was closed with a 4-0 absorbable suture by subcuticular methods consecutively. (Figure 9). 


\section{Data Collection}

The patients' age, sex, body mass index, American Society of Anesthesiologists (ASA) score, site of hernia, operative time, bleeding volume, conversion, postoperative hospital stay, follow-up duration, complications, and hernia recurrence were recorded. All of these patients were followed up by phone calls. The results are expressed as means \pm SD or numbers (percentages).

\section{Results}

SILS-TEP hernia repair was successfully completed in 39 patients. One need an additional ports to complete totally extraperitoneal hernioplasty because the extension of the inferior epigastric artery was detached down from the abdominal wall which influence the range of visibility . Patient demographics and hernia characteristics are summarized in Table 1. The overall mean age

Table 1. Patient demographics and hernia characteristcs

\begin{tabular}{ll}
\hline Variable & Value \\
\hline Number of patients & 40 \\
Male & 36 \\
\hline Female & 4 \\
\hline Mean age,y(range) & $53.8 \square 27-85 \square$ \\
\hline Mean BMI,kg/m²(range) & $21.8(17.2-26.1)$ \\
\hline Median ASA (range) & $1.1(1-2)$ \\
\hline Site of herias(40 patients) & - \\
\hline Right & 19 \\
\hline Left & 14 \\
\hline Both & 7 \\
\hline Type of hernias(n=47) & - \\
\hline Indirect hernia & 36 \\
\hline Direct hernia & 9 \\
\hline Femoral hernia & 2 \\
\hline Scrotal hernia & 6 \\
\hline Recurrent hernia & 0 \\
\hline Incarcerated hernia & 0 \\
\hline
\end{tabular}

\section{BMI,body mass index}

Table 2 Summary of operative and post-operative data 


\begin{tabular}{ll}
\hline Variable & Value \\
\hline Operative time, min(range) & --- \\
Indirect & $73.8(45-130)$ \\
\hline Direct and Femoral & $55.2(34-95)$ \\
\hline Both & $92.3(65-150)$ \\
\hline Conversion to conventional TEP repair & 1 \\
\hline Peritoneum broken accidentally & 13 \\
\hline Hernial sac amputation & 5 \\
\hline Spermaduct injury & 0 \\
\hline Spermatic cord blood vessels injury & 1 \\
\hline Inferior epigastric vessel injury & 0 \\
\hline Corona mortis bleeding & 0 \\
\hline Seroma & 3 \\
\hline Hematoma & 0 \\
\hline Delirium & 0 \\
\hline Chronic pain & 5 \\
\hline Testicular atrophy & 0 \\
\hline Urinary retension & 0 \\
\hline Umbilical hernia & 0 \\
\hline recurrence & 0 \\
\hline Mean postoperative hospital stay(day) & 0 \\
\hline
\end{tabular}

\section{TEP, total extrapeirtoneal}

of the patients was 53.8 years (range, 27 to 85 years) and mean body mass index was $21.8 \mathrm{~kg} / \mathrm{m} 2$ (range, 17.2 to $26.1 \mathrm{~kg} / \mathrm{m} 2$ ). Operative and post-operative data are summarized in Table 2. Mean operative time was 73.8 minutes (range, 45 to 130 minutes) and 55.2 minutes (range, 34 to 95 minutes) in unilateral Indirect hernia and in unilateral Direct or Femoral hernia, respectively . Mean operative time was 92.3 minutes (range, 65 to 150 minutes) in bilateral hernia. One patient experienced spermatic cord blood vessels injury, there is no discomfort during the following 6 months. All of the surgical procedures were completed uneventfully but 13 cases with peritoneum broken and 5 cases with distal sac of indirect hernia amputated. No other intraoperative complication exit. 3 seroma and 5 chronic pain were seen during the 1-6 months follow-up periods, all were treated conservatively. No other major complications, such as mesh infection, testicular atrophy, umbilical hernia, delirium or urinary retension, were noted 
during the follow-up period. There was no mortality or recurrence. Mean post-operative hospital stay was 2.6 days.

\section{Discussion}

Laparoscopic Total extraperitoneal herniorrhaphy (TEP) is an ideal method for inguinal hernia repair. TEP is difficult because it requires preliminary establishment of the preperitoneal space before access to the lens and instruments for specific surgical approaches and tissue levels. Beginners are prone to rupture of peritoneum in the process of dissection due to unclear tissue layers, making the operation difficult or conversion to Transabdominal preperitoneal (TAPP) hernia repair. SILS-TEP has advantages and disadvantages due to the simultaneous operation of lens and Laparoscopic instrument through a single umbilical incision. Since 2013, the author has used A self-made laparoscopic single-incision sealing device which named Iconport to complete various surgeries including transumbilical single-port laparoscopic appendectomy, cholecystectomy, gastroduodenal ulcer perforation repair, and Transabdominal preperitoneal (TAPP) hernia repair successively, with more than 200 cases. During these operations, the abdominal wall tissue adjacent to the umbilicus is cut open, the tissue under the linea alba which composed of peritoneum, preperitoneal fat and preperitoneal fascia could be seen, it seemed that the tissue were thick in $70-80 \%$ of patients but thin and prone to damage in $20-30 \%$ of patients. Based on the understanding of the incision in the umbilical abdominal wall and the anatomical of the preperitoneal space in Total extraperitoneal herniorrhaphy, the author attempted to perform TEP surgery via umbilical incision under posterior rectus abdominis sheath .Among these 40 surgeries, 39 patients successfully completed the SILS-TEP, and the other one added an auxiliary operating hole and completed the TEP approach. In our method, we believe that the key to the SILS-TEP operation is that the laparoscopic Iconport single-port device which used in the surgery. The silicone seal sleeve of Iconport which entering the incision only needs a small preperitoneal space to establish the pneumoperitoneum for laparoscopic operation. Because the initial preperitoneal space required for pneumoperitoneum is relatively small, it is less likely to cause peritoneal rupture under the incision or intraperitoneal hemorrhage. Once the pneumoperitoneum is successfully established, it becomes relatively easy to expand the preperitoneal space with the cooperation of two laparoscopic surgical instruments in the view of the microscope under the condition of maintaining good tension. As previously reported, most of the operations of SILS-TEP were performed in the anterior space of the posterior rectus sheath of the hernia side, which has the advantage that the peritoneum adjacent to the incision is not easy to be damaged. The author found it is more reasonable to creat the preperitoneal space under the posterior sheath of the rectus abdominis via the umbilical incision ,especially in the operation of bilateral hernia because it is not necessary to cut the posterior sheath of the rectus abdominis at the semi-loop line. In the surgery of SILS-TEP, two 
laparoscopic surgical instruments always enter the operative field through the same incision with the lens, and one instrument always presses down on the peritoneum, so the vision in front of the lens is always guaranteed even if the peritoneum is damaged at the beginning of the operation. Peritoneal rupture may delay the process of surgery but will not result in the transition to TAPP surgery. Hem-o-Lok clamping or stitching can be used to repair the peritoneal rupture far from the incision, while the peritoneal rupture near the incision can be left untreated because it is far from the mesh area of hernia repair. If the peritoneum is damaged near the incision, the lens and laparoscopic instruments can be used to observe the peritoneal cavity or assist in the operation of hernia contents retraction. Therefore, the author believes that during the surgery of SILS-TEP, the peritoneal cavity can be explored like TAPP surgery, and such a strategy is conducive to the operation of complex conditions. The peritoneum can be cut open at the incision, and the instruments can be guided into the peritoneal cavity or the preperitoneal space by the laparoscope, and then the instruments can push aside the peritoneum to create conditions for the follow-up of the lens. Most surgeons would believe a single incision laparoscopic operation is difficulty. In fact, the operation of expanding the preperitoneal space in the SILS-TEP is basically one instrument pulling or pressing the peritoneum to assist in the exposure of the operative field, and the other instrument to complete the tissue separation. In this operation, there is no problem in the flexibility of operation because only one instrument is frequently moving. When the indirect hernia sac is dissected, two adjacent operating holes on the panel can be selected. In this way, the two laparoscopic instruments will enter the preperitoneal space from a nearly parallel angle, and tip of the two instruments can be flexibly cooperated alternately. There are four operation holes on the panel of Iconport single-incision sealing device. Usually, the lens enters through the middle operation hole. Two operation holes on both sides of the lens or two adjacent operation holes are selected to operate, which represent two manipulative methods respectively. When a manipulative method is difficult, another method of manipulation can often be an effective replacement. Therefore, the panel features of Iconport are the basis for the flexible operation of laparoscopic surgery. During the operating of single-incision laparoscopic surgery, two laparoscopic instruments perform different functions, one pull or press organizations to create conditions for operation, which need a lever fulcrum to economize labour, the other one as the main operating instrument need flexible operation. The panel of the Iconport is made of hard material, the diameter of the operating hole is set at $15 \mathrm{~mm}$ to ensure that the laparoscopic instrument with a diameter of $5 \mathrm{~mm}$ has enough inclination angle in the operating hole. By selecting different operating hole to cooperate with each other, the operator can avoid the blind area of operation, the side wall of the operation hole is the fulcrum of instrument, which help the performer operation more efficient. When the position relationship between the lens and the instrument which responsible for pulling the tissue has been determined, there is a relatively loose clearance in the incision for the main 
operating hole instrument to use, when the performer insert the instrument in the right space ,the operating must be flexible. Therefore, the operation panel of Iconport not only provide stable and laborsaving fulcrum for the lens and operating instrument, but also make the instrument in the main operating hole move flexibly. The Iconport single-incision sealing device has the advantage of flexibility. In this study, the author chose ETHICON ULTRAPRO $15 * 17 \mathrm{~cm}$ Mesh as hernia repair material, because in the process of SILS-TEP surgery, the preperitoneal space was extended from navel, the Retzius' space is usually exposed throughout the pubic symphysis, full exposure of Retzius' space is conducive to maintaining peritoneal integrity when entering Bogros' space. However, for unilateral hernia, there is a risk of the mesh shifting inward, so the author chose the relatively large mesh currently available on the market. In bilateral hernia repair, the mesh overlaps more in the midline, which is also conducive to the coverage of the most common area of direct hernia. In summary, the author believes that relatively larger hernia repair materials should be selected in the operation of SILS-TEP to reasonably cover the preperitoneal space created during the operation.

\section{Conclusion}

The author believes that the operation of SILS-TEP via umbilical incision under posterior rectus abdominis sheath is safe and feasible. The Iconport single-port device has potential advantages in SILSTEP surgery.

\section{Abbreviations}

SILS-TEP , single-incision laparoscopic surgery totally extraperitoneal

BMI,body mass index

TEP,total extrapeirtoneal

\section{Declarations}

- Ethics approval and consent to participate This study has been reviewed by the research ethics committee of the Affiliated Hospital of Medical School of Ningbo University. Reference Number:2019KY0402.

- Consent for publication All information in this study was approved for publication by all participants.

- Availability of data and material The datasets used and/or analysed during the current study are available from the corresponding author on reasonable request.

- Competing interests The authors declare that they have no competing interests. 
- Funding No financial support for this study.

- Authors' contributions The author(Xiaojun Wang) is the inventor of the self-made single port device named Iconport. This article is the initial experience with Iconport. All authors participated in the study. All authors read and approved the final manuscript.

- Acknowledgements The authors would like to acknowledge the leader of the Affiliated Hospital of Medical School of Ningbo University for supporting this study.

\section{References}

1. Jaksa Filipovic-Cugura,Iva Kirac,Tomislav Kulis etal. Single-incision laparoscopic surgery (SILS) for totally extraperitoneal (TEP) inguinal hernia repair: first case. Surg Endosc (2009) 23:920-921

2. Jaksa Filipovic Cugura, Iva Kirac, Tomislav Kulis, etal. Comparison of Single Incision Laparoscopic Totally Extraperitoneal and Laparoscopic Totally Extraperitoneal Inguinal Hernia Repair: Initial Experience. Endourol. 2012 Jan;26(1):63-66.

3. S. Agrawal,Shaw,Y. Soon. Single-port laparoscopic totally extraperitoneal inguinal hernia repair with the TriPort system: initial experience. Surg Endosc (2010) 24:952-956

4. Ji Hoon Kim, Seung Man Park, Jin Jo Kim, etal. Initial experience of single port laparoscopic totally extraperitoneal hernia repair: nearly-scarless inguinal hernia repair.J Korean Surg Soc 2011(81):339-343.

5. JiHoonKim,YoonSukLee,JinJoKim etal. Single Port Laparoscopic Totally Extraperitoneal Hernioplasty: A Comparative Study of Short-term Outcome with Conventional Laparoscopic Totally Extraperitoneal Hernioplasty. World J Surg (2013) 37:746-751

6. H.Kim C.H.An Y.S.Lee.Single incision laparoscopic totally extraperitoneal hernioplasty (SIL-TEP): experience of 512 procedures. Hernia (2015) 19:417-422.

7. B.J.Choi1,W.J.Jeong1,I.K.Lee2, etal. Single-port versus conventional three-port laparoscopic totally extraperitoneal inguinal hernia repair: a randomized controlled trial.Hernia (2016) 20:789-795.

8. Yoon Dae Han, Seungwan Park, Woo Ram Kim, etal. Safety and Efficacy of Single-Incision Laparoscopic Totally Extraperitoneal Inguinal Hernia Repair: Comparative Study with Conventional Laparoscopic Totally Extraperitoneal Inguinal Hernia Repair.Journal of Laparoendoscopic \& Advanced Surgical Techniques(2017)27:1-5.

9. Masaki Wakasugi, Toru Masuzawa,Mitsuyoshi Tei , etal. Single-incision totally extraperitoneal inguinal hernia repair: our initial 100 cases and comparison with conventional three-port laparoscopic totally extraperitoneal inguinal hernia repair.Surg Today (2015) 45:606-610.

10. Masaki Wakasugi, Mitsuyoshi Tei, ,Hiroki Akamatsu. Single-Incision Totally Extraperitoneal Inguinal Hernia Repair After Previous Inguinal Hernia Repair. Surg Laparosc Endosc Percutan Tech(2016) 26:149-152. 
11. Masaki Wakasugi, Mitsuyoshi Tei, Yozo Suzuki, etal. Single-incision totally extraperitoneal inguinal hernia repair is feasible and safe in patients on antithrombotic therapy: A single center experience of 92 procedures. Asian $\mathrm{J}$ Endosc Surg (2017) 10:301-307.

12. Masaki Wakasugi · Yozo Suzuki · Mitsuyoshi Tei, etal. The feasibility and safety of single incision totally extraperitoneal inguinal hernia repair after previous lower abdominal surgery: 350 procedures at a single center. Surg Today (2017) 47:307-312.

13. Masaki Wakasugi, Yujiro Nakahara, Masaki Hirota, etal. Learning curve for single-incision laparoscopic totally extraperitoneal inguinal hernia repair. Asian J Endosc Surg (2018):1-5.

14. Wang,XJ, Zhang,YZ, Shuai YF, etal. Application of Self-made Single Port Device for Laparoscopic Appendectomy: a Report of 30 Cases. Chinese journal of minimally invasive surgery, 2014,14(9):837838.

15. Zhang,YZ, Wang,XJ, Shuai,YF, etal. Transumbilical Single-port Laparoscopic Cholecystectomy by Using a Self-made Device Iconport: a Report of 50 Cases. Chinese journal of minimally invasive surgery,2015,15(6):543-545.

\section{Figures}




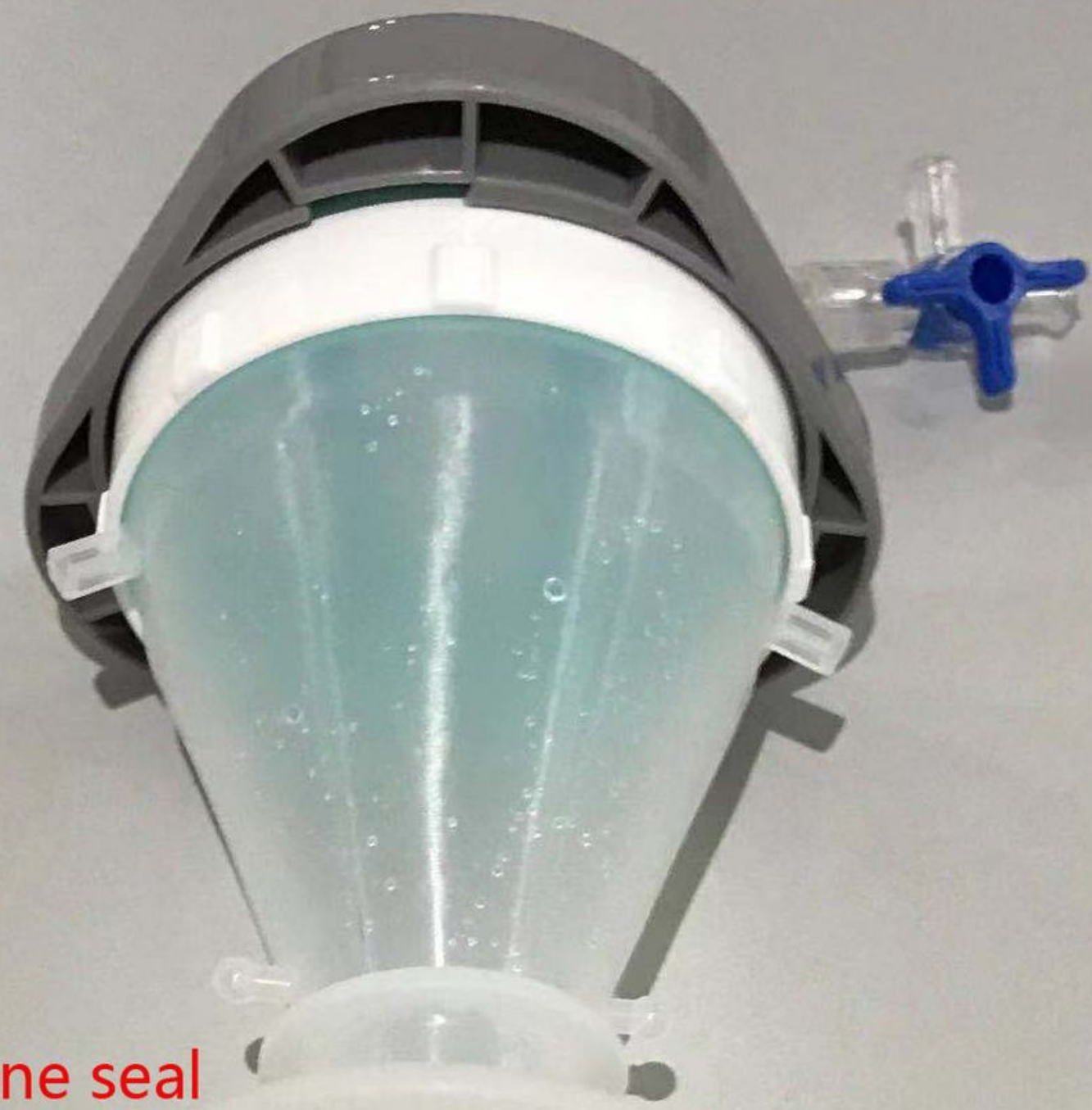

Silicone seal

sleeve $\rightarrow$

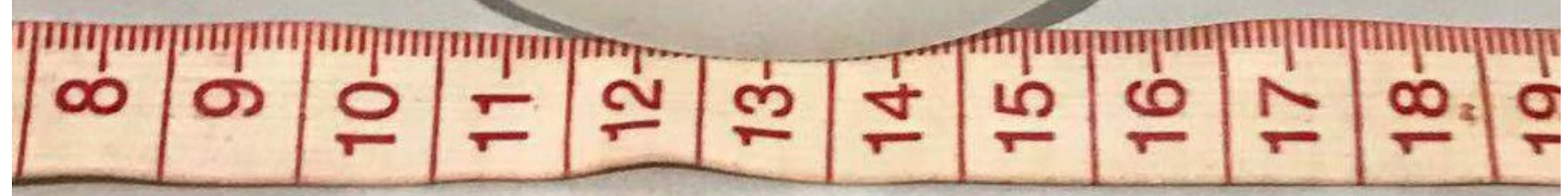

\section{Figure 1}

Single- incision sealing device which named Iconport(underside) 


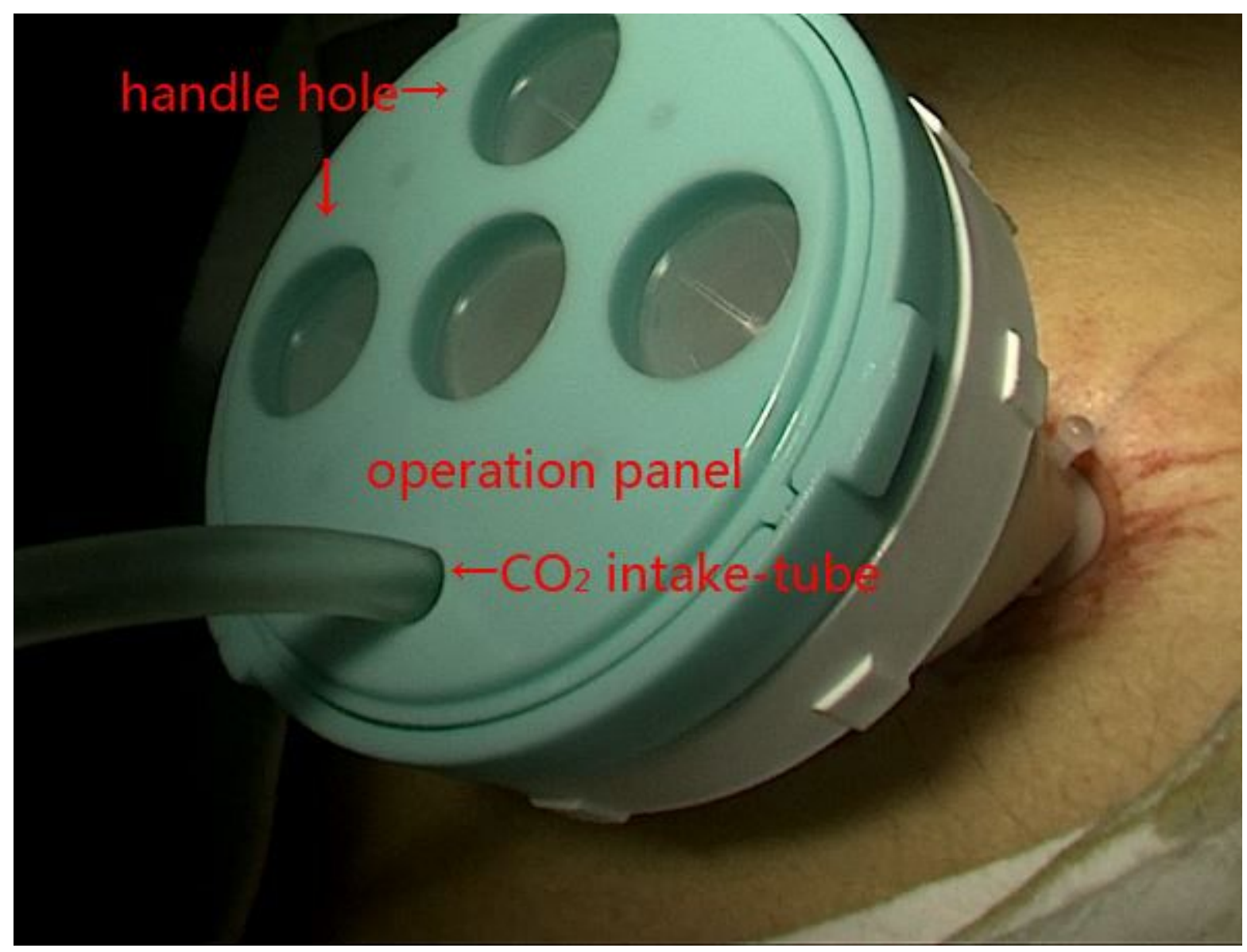

\section{Figure 2}

Single- incision sealing device and navel incision

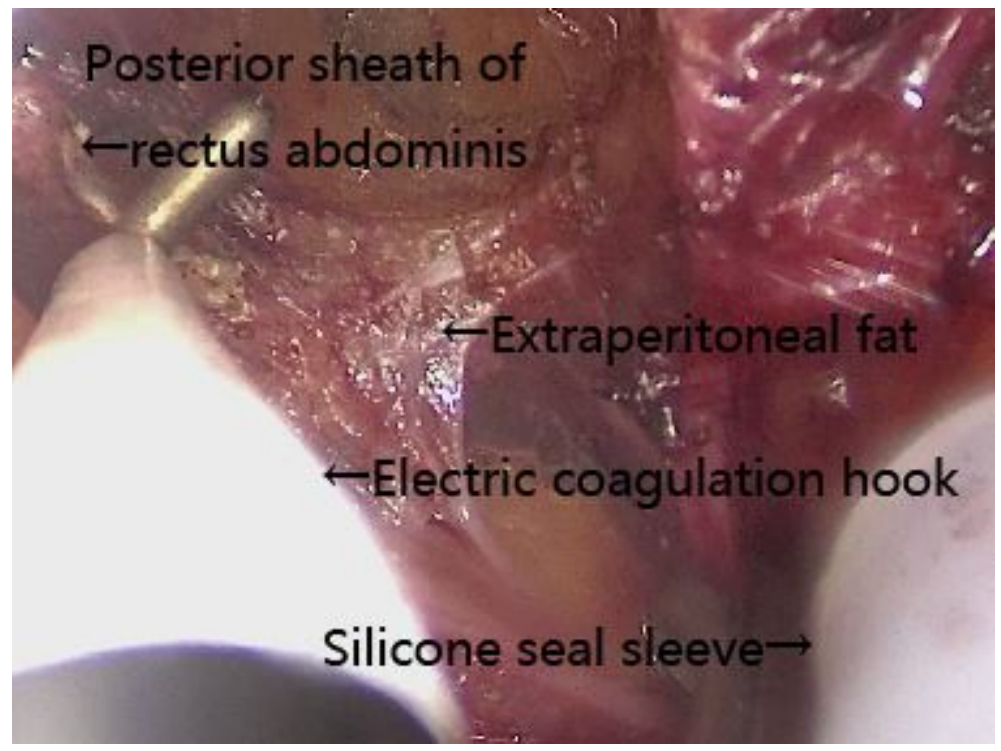

Figure 3

Peritoneal space under posterior rectus abdominis sheath in umbilical incision 


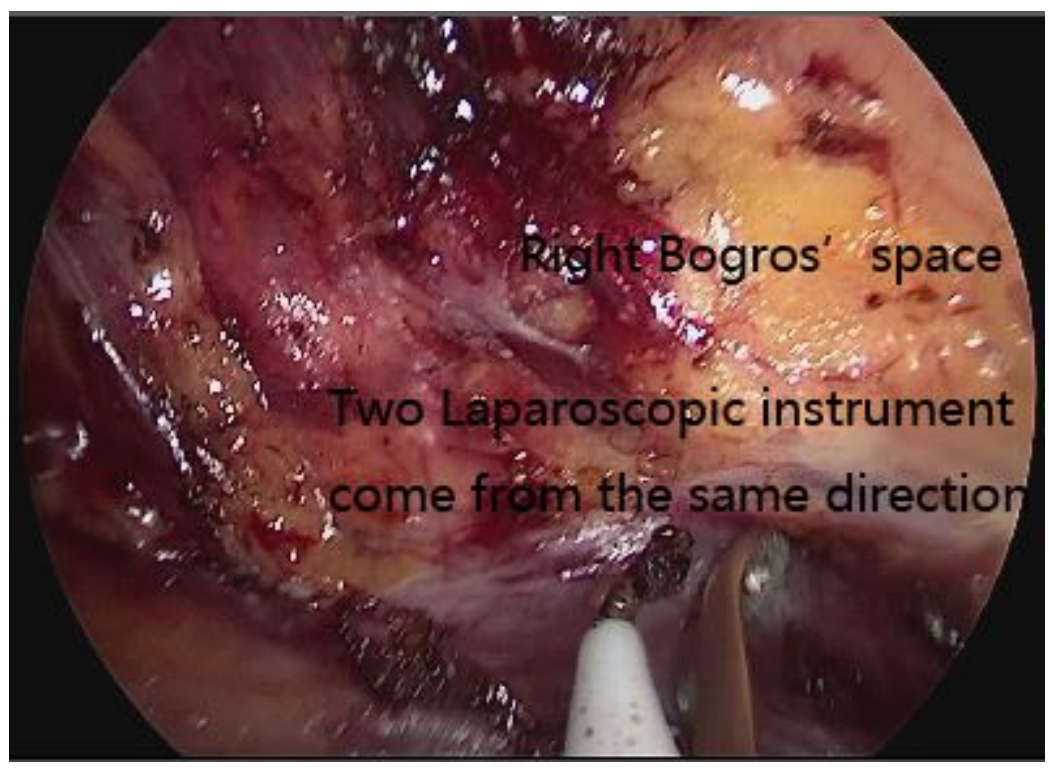

Figure 4

Retzius' space revealed initially

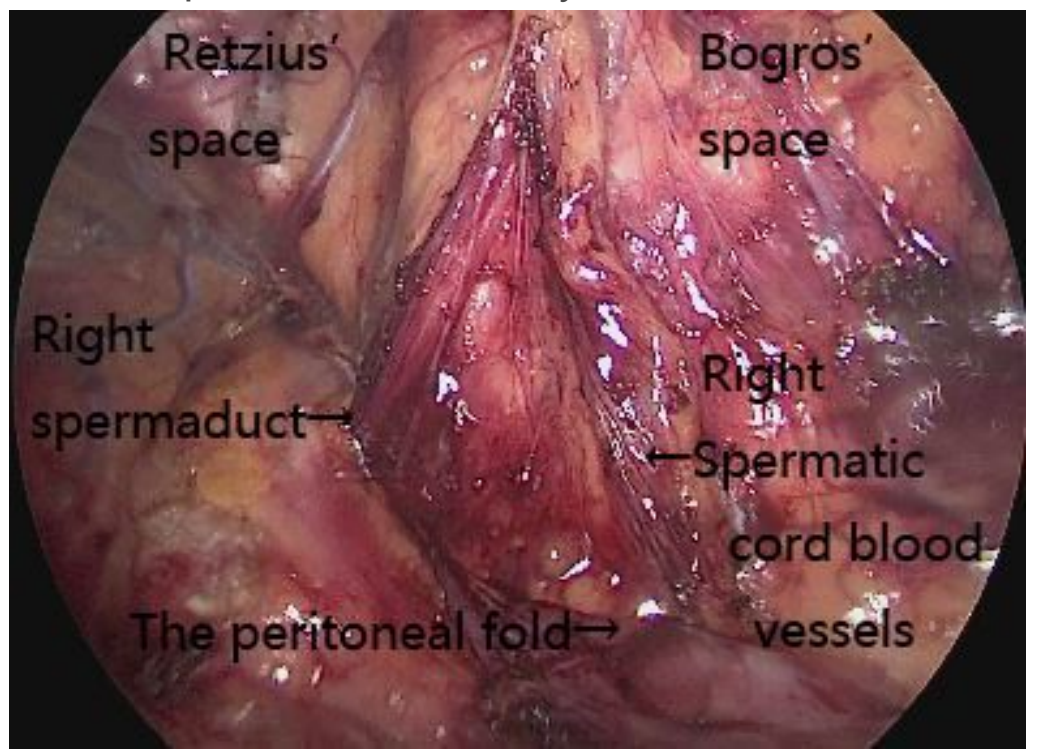

Figure 5

Intraoperative laparoscopic view of the preperitoneal space (right) 


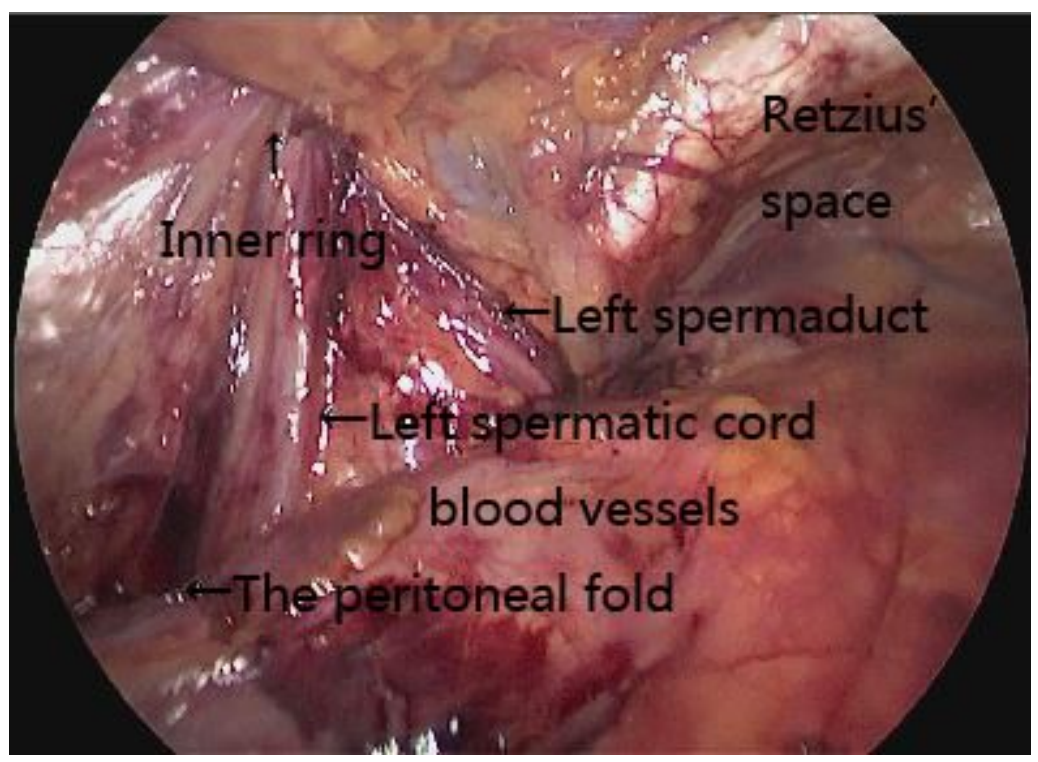

\section{Figure 6}

Intraoperative laparoscopic view of the preperitoneal space (left)

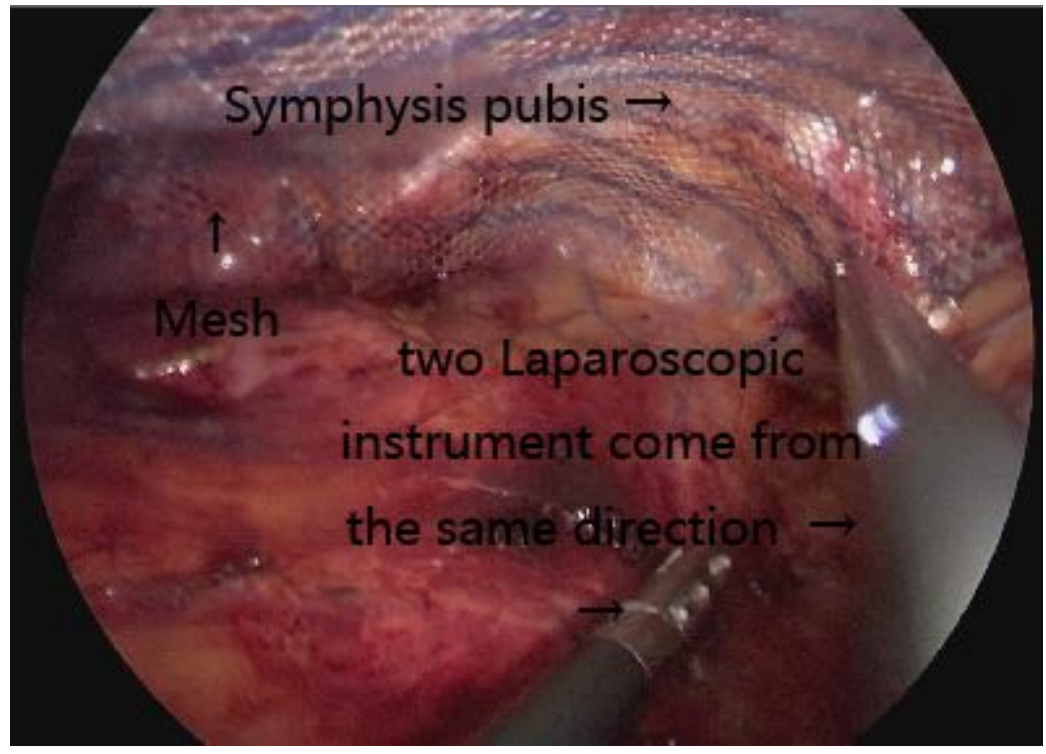

\section{Figure 7}

Mesh deployed to cover the whole myopectineal orifice 


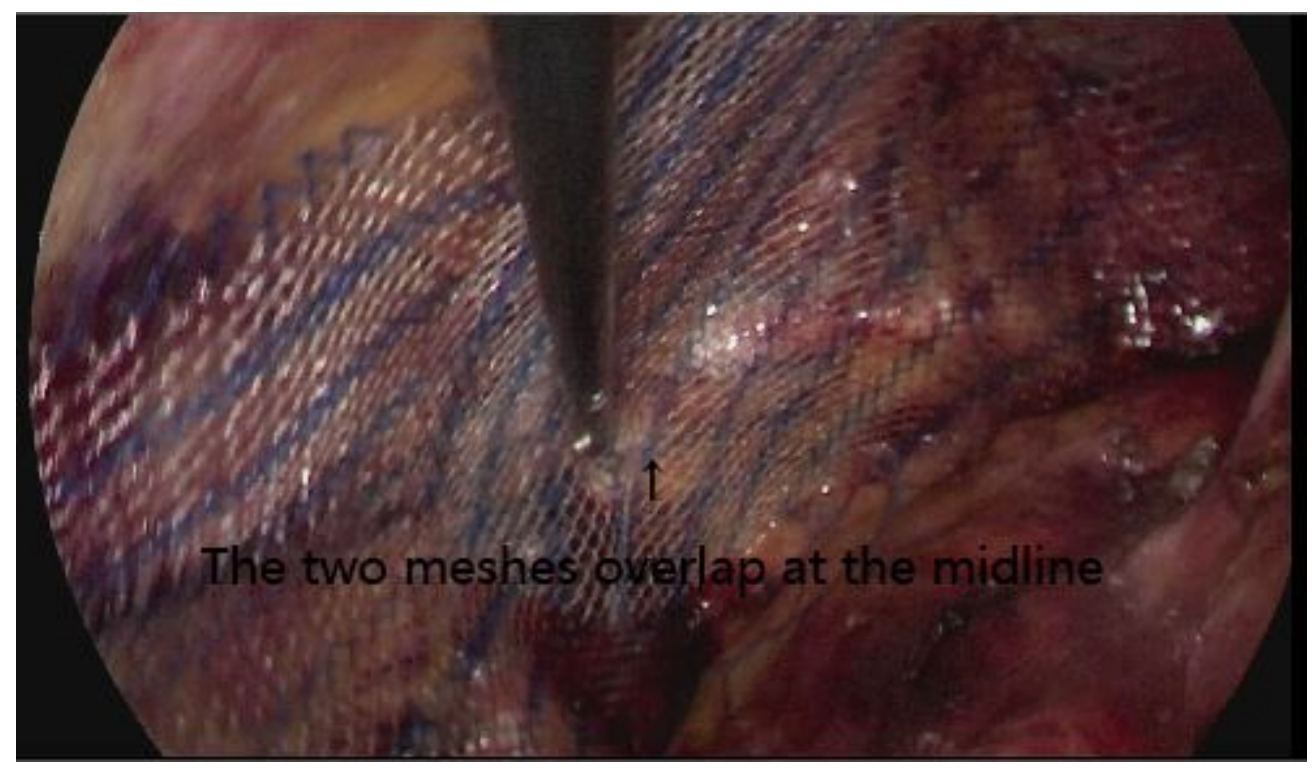

Figure 8

Bilateral mesh overlaps and crosses at the midline

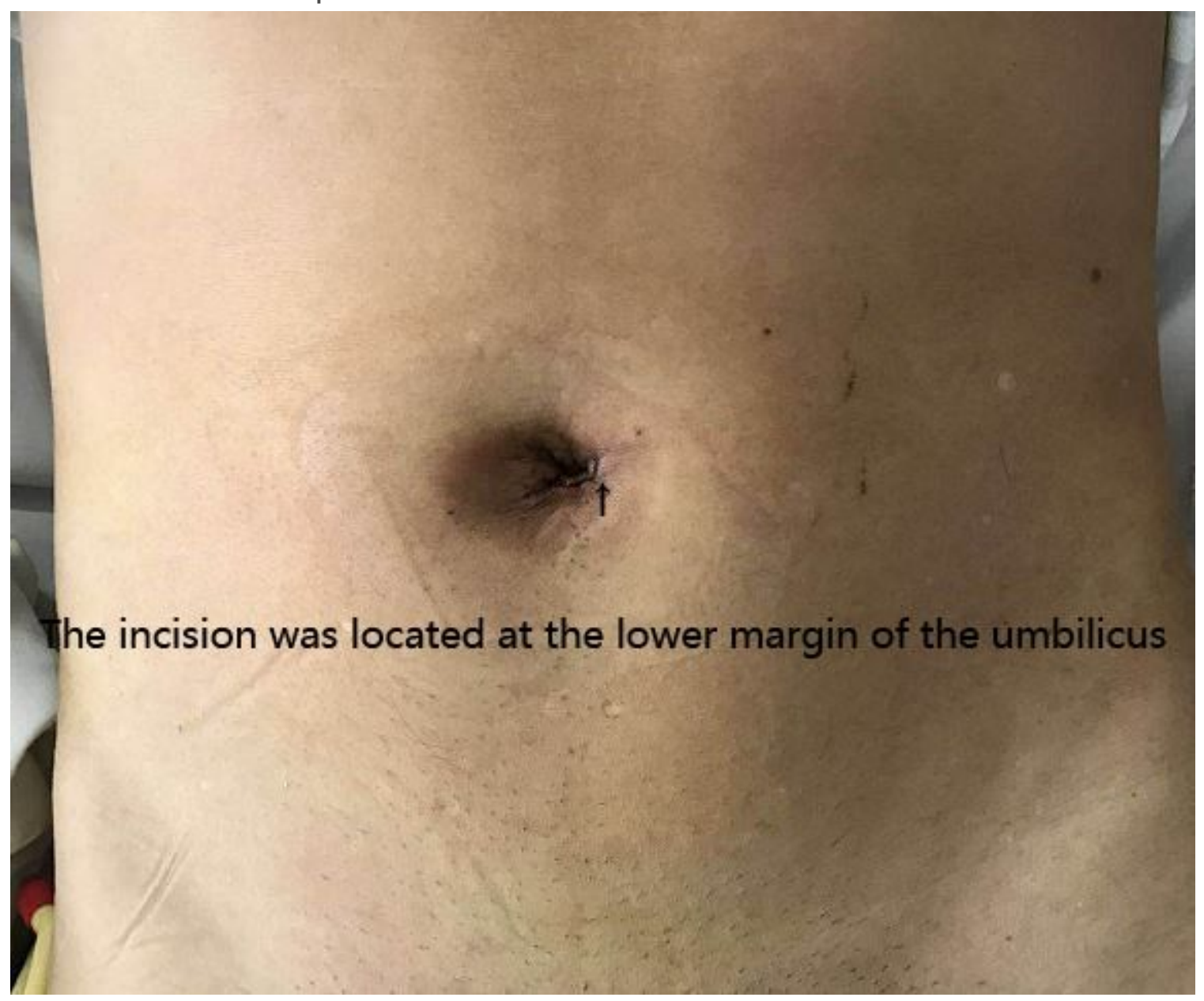

Figure 9

Photograph of postoperative incision 